statistically significant difference in risk factors for raised DL comparing age, bowel resection, absence of ileo-caecal valve, abnormalities on barium study and use of proton pump inhibitors. SBL was significantly shorter $(p=0.001)$ in raised DL group (median $29.6 \%$; range 11.4-100) than in group without (median 100\%; range 19.10 100). Patients with $<35 \%$ SBL, had $77 \%$ sensitivity for developing raised DL. Relationship to feed could not be analysed due to lack of accurate information on patients' carbohydrate intake. Response to treatment was available in $12 / 25$ and all had improvement in symptoms with fall in DL. Recurrence occurred in $48 \%$.

Conclusion Children with IF due to $<35 \%$ expected SBL, when screened, have a $77 \%$ likelihood of having SBBO shown by raised $\mathrm{DL}$. Screening in at risk patients allows prompt detection and treatment of SBBO. Recurrence is common necessitating prolonged antibiotic regimens.

\section{G197(P) HEPATIC HAEMANGIOMA AND CONJUGATED HYPERBILIRUBINEMIA - A CASE REPORT}

doi:10.1136/archdischild-2013-304107.209

'ND Ruth, 'J Kirk, 'D Kelly. 'Liver Unit, Birmingham Children's Hospital, Birmingham, UK; ${ }^{2}$ Dept of Endocrinology, Birmingham Children's Hospital, Birmingham, UK

Background Infantile hepatic haemangioma, the most common benign vascular tumour of the liver in childhood, presents within the first months of life. $80 \%$ present in infancy and nearly half have associated cutaneous hemangiomas. Other extrahepatic lesions may also be present including pulmonary and cerebral haemangiomata.

Subjects and Methods A term neonate presented with respiratory distress, unstable blood sugars and was small for gestational age. She was referred to a liver unit for management of hepatic haemangioma.

Results We describe a neonate who presented with hepatic haemangioma, cardiac failure and conjugated hyperbilirubinemia which was due to hypopituitarism. This combination of clinical disease has not previously been reported. The diagnosis of hypopituitarism was considered because the infant had low blood sugars with prolonged conjugated jaundice during the initial assessment and treatment. Although jaundice is associated with large hepatic haemangiomata it is generally unconjugated unless there is a degree of biliary obstruction associated with the size of the haemangioma. Following diagnosis of hypopituitarism, commencement of replacement therapy with hydrocortisone and thyroxine resulted in resolution of symptoms and stabilisation of her condition.

Conclusion This is an unusual presentation of hypopituitarism, and could have been overlooked in view of the other pathology present with adverse consequences for her future health and development.

\section{G198(P) PLASMA ARGININE LEVELS AND BLOOD GLUCOSE CONTROL IN VERY PRETERM INFANTS RECEIVING TWO DIFFERENT PARENTERAL NUTRITION REGIMENS}

doi:10.1136/archdischild-2013-304107.210

${ }^{1} \mathrm{~L}$ Burgess, ${ }^{1} \mathrm{C}$ Morgan, ${ }^{2} \mathrm{~K}$ Mayes, ${ }^{3} \mathrm{M}$ Tan. 'Department of Neonatology, Liverpool Women's Hospital, Liverpool, UK; ${ }^{2}$ Department of Clinical Chemistry, Alder Hey Children's Hospital, Liverpool, UK; ${ }^{3}$ Department of Paediatrics, Alder Hey Children's Hospital, Liverpool, UK

Background and Introduction We have previously shown that improving early protein intake is associated with a reduction in insulin-treated hyperglycaemia in preterm infants $<29$ weeks gestation. The effect of amino acids (AA) on insulin secretion is well described in preterm infants with arginine recognised as a potent secretagogue. We hypothesised that low arginine levels would be
Abstract G198(P) Table 1 Mean (SE) blood glucose (mmol/l; 5 day time periods) and insulin use (total days, d1-15)

\begin{tabular}{lllllllll}
\hline Group & $\mathbf{d 1 - 5}(\mathbf{C})$ & $\mathbf{d 6}-10(\mathbf{C})$ & $\mathbf{d 1 1 - 1 5}(\mathbf{C})$ & Insulin & $\mathbf{d 1 - 5 ( H )}$ & $\mathbf{d 6 - 1 0 ( H )}$ & $\mathbf{d 1 1 - 1 5 ( H )}$ & Insulin \\
\hline lowARG & $6.9(0.3)$ & $8.6(0.4)$ & $7.1(0.4)$ & 110 & $8.2(0.4)$ & $9.9(0.4)$ & $7.6(0.4)$ & 203 \\
highARG & $6.6(0.6)$ & $7.3(0.4)$ & $6.0(0.5)$ & 30 & $6.7(0.4)$ & $8.3(0.6)$ & $6.6(0.4)$ & 66 \\
p value & 0.58 & $<0.05$ & 0.11 & & $<0.01$ & 0.02 & 0.11 &
\end{tabular}

associated with an increase in insulin-treated hyperglycaemia and higher mean daily blood glucose levels (day1-15) in infants born $<29$ weeks gestation.

Methods We performed a secondary analysis on previous randomised controlled trial data comparing hyperalimentation $(\mathrm{H})$ and control (C) regimens. The hyperalimentation regimen provided $20 \%$ more carbohydrate than the control regimen. Daily carbohydrate and protein intake data and mean daily blood glucose and insulin use data from the first 15 days of life were substratified according to high (highARG) or low (lowARG) arginine levels on day 8-10 using a reference population based median plasma level (57 micromol/1).

Results In group C, substratification identified 41 lowARG and 19 highARG infants. There were no differences in basic demographic factors, carbohydrate or protein intake. Hyperglycaemia peaked on day 5-10. Low arginine levels were associated higher mean daily blood glucose levels (day 6-10) and more insulin treatment (Table 1; group $\mathrm{C}$ ). In group $\mathrm{H}$, substratification identified 33 lowARG and 22 highARG infants. LowARG infants were of lower gestation and birthweight $(p<0.01)$ There were no differences in carbohydrate or protein intake. Low arginine levels were associated higher mean daily blood glucose levels (day 1-5, 6-10) and more insulin treatment (Table 1; group $\mathrm{H}$ ).

Conclusion Low plasma arginine levels in very preterm infants are associated with poorer blood glucose control.

\section{G199(P) USE OF FISH-OIL BASED INTRAVENOUS LIPID EMULSION AS A RESCUE IN INFANTS WITH INTESTINAL FAILURE- ASSOCIATED LIVER DISEASE WHO DEVELOP SEPSIS}

doi:10.1136/archdischild-2013-304107.211

${ }^{1} \mathrm{HM}$ Lee, ${ }^{2} \mathrm{~A}$ Hickey, ${ }^{3} \mathrm{M}$ O'Meara, ${ }^{3} \mathrm{~L}$ Thompson, ${ }^{1} \mathrm{~J}$ Hind. ${ }^{1}$ Paediatric Hepatology, King's College Hospital, London, UK; ${ }^{2}$ Paediatrics, King's College Hospital, London, UK; ${ }^{3}$ Pharmacy, King's College Hospital, London, UK

Aims In infants with intestinal failure-associated liver disease (IFALD), it is known that episodes of sepsis can be accompanied by a significant deterioration in liver function. We hypothesised that an intravenous lipid emulsion (ILE) comprised solely of fish oil, high in omega-3 fatty acids, such as Omegaven ${ }^{\circledR}$, may protect the liver in these infants during episodes of sepsis. Our aim is to describe the potential role for Omegaven ${ }^{\circledR}$ as a rescue therapy in infants with sepsis and established IFALD.

Methods A mixed source ILE containing both omega-3 and omega-6 fatty acids (SMOFlipid ${ }^{\circledR}$ ) was used as first-line in infants at high risk of IFALD. When infants with IFALD developed sepsis, Omegaven ${ }^{\circledR}$ was used as the sole ILE for up to 14 days. A retrospective review of their case notes was conducted.

Results Omegaven ${ }^{\circledR}$ was well tolerated in all infants. 7 infants had Omegaven ${ }^{\circledR}$ treatment during a 14-month period (August 2011-October 2012). Median birth weight was 1000g (range 5271870). Median gestation at birth was 30 weeks (range 24-34). Of the 7 patients, 2 had gastrochisis and 5 had necrotising enterocolitis (NEC). One patient with gastrochisis developed NEC. 2 patients were late transfers at 4-5 months of age from other hospitals with severe and progressive IFALD. Both subsequently died. Median age at start of Omegaven ${ }^{\circledR}$ was 63 days (range 7-189). 3 patients did not 